\title{
Improving surface acousto-optical interaction by high aspect ratio electrodes
}

\author{
Dühring, Maria Bayard; Laude, Vincent; Khelif, Abdelkrim
}

Published in:

Journal of Applied Physics

Link to article, DOI:

$10.1063 / 1.3266017$

Publication date:

2009

Document Version

Publisher's PDF, also known as Version of record

Link back to DTU Orbit

Citation (APA):

Dühring, M. B., Laude, V., \& Khelif, A. (2009). Improving surface acousto-optical interaction by high aspect ratio electrodes. Journal of Applied Physics, 106(11), 113518. https://doi.org/10.1063/1.3266017

\section{General rights}

Copyright and moral rights for the publications made accessible in the public portal are retained by the authors and/or other copyright owners and it is a condition of accessing publications that users recognise and abide by the legal requirements associated with these rights.

- Users may download and print one copy of any publication from the public portal for the purpose of private study or research.

- You may not further distribute the material or use it for any profit-making activity or commercial gain

- You may freely distribute the URL identifying the publication in the public portal

If you believe that this document breaches copyright please contact us providing details, and we will remove access to the work immediately and investigate your claim 


\title{
Improving surface acousto-optical interaction by high aspect ratio electrodes
}

\author{
Maria B. Dühring, ${ }^{1, a)}$ Vincent Laude, ${ }^{2}$ and Abdelkrim Khelif ${ }^{2}$ \\ ${ }^{1}$ Department of Mechanical Engineering, Solid Mechanics, Technical University of Denmark, $2800 \mathrm{Kgs}$. \\ Lyngby, Denmark \\ ${ }^{2}$ Institut FEMTO-ST, Université de Franche-Comté, CNRS, ENSMM, UTBM, 32 Avenue de l'Observatoire, F- \\ 25044 Besançon, France
}

(Received 27 July 2009; accepted 27 October 2009; published online 8 December 2009)

\begin{abstract}
The acousto-optical interaction of an optical wave confined inside a waveguide and a surface acoustic wave launched by an interdigital transducer (IDT) at the surface of a piezoelectric material is considered. The IDT with high aspect ratio electrodes supports several acoustic modes that are strongly confined to the surface, causing a significant increase in the strain underneath the surface. A finite element method is employed to model the surface acoustic waves generated by a finite length IDT with 12 electrode pairs and subsequently to study their interaction with an optical wave propagating in a waveguide buried in the lithium niobate substrate supporting the electrodes. The interaction can be increased up to 600 times using these new types of surface acoustic waves as compared to using a conventional IDT with thin electrodes. This result could find applications in improved acousto-optical integrated modulators. (C) 2009 American Institute of Physics.
\end{abstract}

[doi:10.1063/1.3266017]

\section{INTRODUCTION}

This paper is concerned with surface acoustic waves (SAWs) generated by high aspect ratio (HAR) electrodes and their use for improving integrated acousto-optical modulators. SAWs are elastic waves that propagate along a material surface with most of their energy density concentrated at the surface. ${ }^{1}$ Two main types of SAWs exist. One type is the Rayleigh wave, which is mainly polarized in the vertical and longitudinal direction, and the other type includes surface waves that are mostly polarized in the shear horizontal $(\mathrm{SH})$ direction, such as the Bleustein-Gulyaev wave or the leaky SAWs of lithium tantalate and lithium niobate. In piezoelectric materials, the elastic field is accompanied by an electric field and SAWs can be generated by interdigital transducers (IDTs) by the inverse piezoelectric effect. SAWs are extensively used in electromechanical filters and resonators for telecommunication as well as in oscillators and sensors. ${ }^{2-4} \mathrm{~A}$ new application is the modulation of optical waves, see Ref. 5 for an overview. In Refs. 6-8, experimental results for a compact and monolithic modulator consisting of a SAW driven Mach-Zehnder interferometer (MZI) are presented. A coupled piezoelectric and optical finite element model is introduced in Ref. 9, which is employed to calculate and improve the acousto-optical interaction in such a modulator by a parameter study of the geometry. In these applications SAWs are generated by IDTs with limited electrode height such that the SAW mode properties are similar to the propagation on a free surface. In Ref. 10, it was shown theoretically by a combined finite element and boundary element method that the SAW properties are significantly changed when HAR electrodes are used instead. Multimode SAW propagation was found and up to a tenfold slowing of the

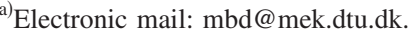

SAW velocity was obtained. Experimental results are presented in Ref. 11 and a fine agreement between numerical and experimental results was found. In order to plot the mode shapes, we introduced a finite element model of a piezoelectric anisotropic material in Ref. 12 to simulate the HAR electrodes utilizing periodic boundary conditions. A perfectly matched layer (PML) was employed at the bottom to absorb the mechanical and electrical disturbances propagating away from the surface. ${ }^{9,13} \mathrm{~A}$ fine agreement was found between this new model and the previous results in Refs. 10 and 11. With this model, it was possible to obtain the mode shape of all the resonances for the periodic structure and it was observed that each mode shape consisted of a combined vibration at the surface and in the electrode. The mechanical energy confinement to the electrodes was calculated and it was observed that it increases with increasing aspect ratio. This indicates that the phase velocity is slowed down because of energy storage in the electrodes.

In this work we modify the periodic model to examine the structure with a finite number of HAR electrodes by employing PMLs at the vertical borders. The resonance frequencies and the energy confinement to the electrodes are compared for the periodic and finite models in order to validate the finite model and a fine agreement is obtained. The finite HAR device is then employed to calculate the acoustooptical interaction in an optical waveguide based on the acousto-optical model from Ref. 9. The two first order optical modes are matched with the first six acoustic modes at the surface where the acoustic strain energy has a high confinement. The acoustic strain is first calculated by the piezoelectric model, which is then coupled to a model of the optical wave. From this coupled model, it is possible to calculate the difference in effective refractive index between the two cases where a negative and a positive electric potential, respectively, are applied to the electrode above the 


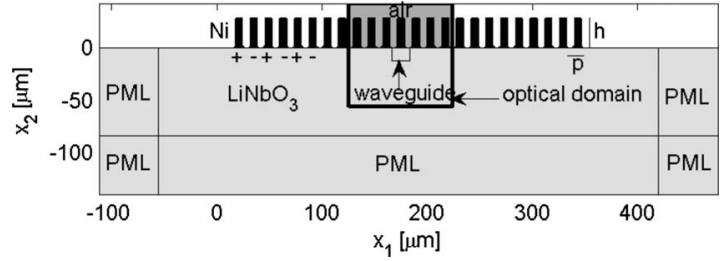

FIG. 1. The geometry of the acousto-optical problem with nickel (Ni) electrodes on a lithium niobate $\left(\mathrm{LiNbO}_{3}\right)$ substrate. The height of the electrodes is $h$ and $p$ is the period of the problem. PMLs absorb the waves at the boundaries and the thick line indicates the optical domain.

waveguide. This is a measure for how efficient each acoustic mode interact with the optical modes. The results of the acousto-optical interaction with the HAR electrodes are compared to similar results for a conventional device with thin electrodes, and it is concluded that the interaction can be increased more than 600 times for the new types of waves.

\section{THE ACOUSTO-OPTICAL MODEL}

The finite model for computing the interaction between SAWs generated by HAR electrodes and an optical wave in a waveguide is described in this section and is similar to the model from Ref. 9.

\section{A. Problem description}

The two-dimensional (2D) computational domain is illustrated in Fig. 1. The electrodes consist of nickel (Ni) and are placed on top of a substrate of $\mathrm{Y}+128^{\circ}$ cut lithium niobate $\left(\mathrm{LiNbO}_{3}\right)$. The width of the electrodes is $a$ and the height is $h$. The parameter $p=2 a$ is the period in the problem and is of the order of a few microns in applications. In fabricated devices, the corners at the interface between the electrodes and the substrate will not be sharp. To imitate that and to avoid stress concentrations in the simulations, the corners have a small rounding, which can be clearly seen in Fig. 5, for instance. The SAW is generated by applying an alternating electrical potential to the HAR electrodes as indicated at the left electrodes in Fig. 1. The metal is assumed to be perfectly conducting such that the electrical potential only needs to be applied at the interface between $\mathrm{Ni}$ and $\left(\mathrm{LiNbO}_{3}\right)$. The SAW then propagates in the left and the right horizontal direction and both the mechanical and electrical disturbances are absorbed in the PMLs. The SAW passes through an optical waveguide in the middle where light propagates out of the plane in the $x_{3}$-direction. To simulate the acousto-optical interaction in the waveguide, a model describing the SAW generation in a piezoelectric material is coupled with an optical model describing the propagation of the optical wave in the waveguide. It is assumed that the strain-optical effect is dominant compared to the electrooptical effect, which will be neglected here. ${ }^{7}$ It is furthermore assumed that the SAW will affect the optical wave, but the optical wave will not influence the SAW, so the problem is solved by first calculating the mechanical strain introduced by the SAW. Then the change in refractive index in the materials due to the strain can be calculated by the strain-optical relation, and finally by solving the optical model the effec- tive refractive index of the different possible light modes can be determined. The practical choice of the metal for the electrodes depends on the combination of electrical and viscoelastic losses, which should both be minimized. The presence of such acoustic low frequency modes is also due to the existence of mechanical resonance of electrodes, which are related to mass density, elastic constant, and geometrical parameters. The technology employed to achieve thick structured electrodes is important. For instance, in Ref. 11, electroplated Ni electrodes were used with success up to 200 $\mathrm{MHz}$ and it is extrapolated that they can be applied to the gigahertz range as well. Heavier metals such as gold could also be considered but have not been tried yet. The mathematical model for the acousto-optical interaction is described in Secs. II B-II D.

\section{B. The piezoelectric model}

The applied electric potential will introduce mechanical displacements in the solid by the inverse piezoelectric effect. The behavior of the piezoelectric material is described by the following model, as found in Ref. 14. A time-harmonic electrical potential,

$$
V\left(x_{j}, t\right)=V\left(x_{j}\right) e^{i \omega t},
$$

with the angular frequency $\omega$ is applied to the electrodes, where $x_{j}$ are the coordinates and $t$ is the time. The mechanical strain $S_{i j}$, assumed small, and the electric field $E_{j}$ are given by the expressions

$$
S_{i j}=\frac{1}{2}\left(\frac{1}{\gamma_{j}} \frac{\partial u_{i}}{\partial x_{j}}+\frac{1}{\gamma_{i}} \frac{\partial u_{j}}{\partial x_{i}}\right) \quad \text { and } \quad E_{j}=-\frac{1}{\gamma_{j}} \frac{\partial V}{\partial x_{j}},
$$

where $u_{i}$ are the displacements. Note that the Einstein notation is not applied in Eq. (2). The parameter $\gamma_{j}$ is an artificial damping at position $x_{j}$ in the PML given by the expression

$$
\gamma_{j}\left(x_{j}\right)=1-i \sigma_{j}\left(x_{j}-x_{l}\right)^{2},
$$

where $x_{l}$ is the coordinate at the interface between the regular domain and the PML and $\sigma_{j}$ is a suitable constant. There is no damping outside the PMLs and $\gamma_{j}$ is here equal to 1 . The mechanical stresses $T_{j k}$ and the electric displacement $D_{i}$ both depend on the strain and the electric fields according to the following constitutive relations:

$$
\begin{aligned}
& T_{j k}=\widetilde{c}_{j k l m}^{E} S_{l m}-\widetilde{e}_{i j k}^{T} E_{i}, \\
& D_{i}=\widetilde{e}_{i j k} S_{j k}+\widetilde{\varepsilon}_{i j}^{S} E_{j},
\end{aligned}
$$

where $\tilde{c}_{j k l m}^{E}$ are the elastic stiffness constants, $\tilde{e}_{i j k}$ are the piezoelectric constants, and $\widetilde{\varepsilon}_{i j}^{S}$ are the permittivity constants. The material tensors are rotated according to Euler's transformation theory ${ }^{15}$ to get the proper piezoelectric directions. This is indicated by the tilde above the material tensors. The two governing equations give the stresses by Newton's second law and the electric displacement from Gauss law:

$$
\frac{1}{\gamma_{j}} \frac{\partial T_{i j}}{\partial x_{j}}=-\rho \omega^{2} u_{i} \quad \text { and } \quad \frac{1}{\gamma_{j}} \frac{\partial D_{j}}{\partial x_{j}}=0
$$

where $\rho$ is the density of the material. The governing equations are second order differential equations for $u_{j}$ and $V$. 
Mechanical and electrical boundary conditions must be specified. Considering the mechanical conditions, the upper surface is stress-free and the vertical sides and the bottom are clamped:

stress free surface: $T_{j k} m_{k}=0$,

clamped surface: $u_{i}=0$,

where $m_{k}$ is the normal unit vector pointing out of the surface. At the upper surface, there are no charges and therefore electric insulation occurs, meaning that the normal component of the electric displacement is zero. At the bottom and to the sides of the domain, it is assumed that the electrical potential is zero, whereas at the interface between the electrodes and the substrate, the potential is $\pm V_{p}$. The electrical boundary conditions are summarized as follows:

electrical insulation: $D_{i} m_{i}=0$,

zero potential: $V=0$,

applied positive potential: $V=V_{p}$,

applied negative potential: $V=-V_{p}$.

The piezoelectric problem is solved by a plane formulation obtained by omitting all derivatives with respect to $x_{3}$. The two governing equations (6) are solved simultaneously to find the four unknowns $u_{1}, u_{2}, u_{3}$, and $V$.

\section{The optical model}

After the mechanical strain in the material has been computed by the piezoelectric model, the refractive index $n_{i j}$ in the strained material can be calculated according to the strain-optical relation: ${ }^{16}$

$$
\Delta b_{i k} b_{k j}=\widetilde{p}_{i j l m} S_{l m},
$$

where $\widetilde{p}_{i j l m}$ are the rotated strain-optical constants and $b_{i k} n_{k j}=\delta_{i j}$. For a given optical mode of order $\nu$ with the propagation constant $\beta_{\nu}$, the effective refractive index $n_{\text {eff, } \nu}$ is defined as

$$
n_{\mathrm{eff}, \nu}=\beta_{\nu} / k_{0},
$$

where $k_{0}$ is the free space propagation constant. It is assumed that the propagating optical modes have harmonic solutions on the form

$$
H_{q, \nu}\left(x_{1}, x_{2}, x_{3}\right)=H_{q, \nu}\left(x_{1}, x_{2}\right) e^{-i \beta_{\nu} x_{3}},
$$

where $H_{q, \nu}$ are the magnetic field components of the optical wave. The governing equations for the magnetic field are the time-harmonic wave equations,

$$
e_{i j k} \frac{\partial}{\partial x_{j}}\left(b_{k l} b_{l m} e_{m p q} \frac{\partial H_{q}}{\partial x_{p}}\right)-k_{0}^{2} H_{i}=0,
$$

where $e_{i j k}$ here is the alternating symbol. For a given value of $k_{0}$, the propagation constant $\beta_{\nu}$ for the possible modes are found by solving the wave equations as an eigenvalue problem, whereby the effective refractive indices are obtained. In this work, the set of equations are reduced such that the model is only solved for the transverse components of the magnetic field $H_{1}$ and $H_{2}$. As the energy of the lower order optical modes is concentrated in the waveguide, the optical domain (where the eigenvalue problem is solved) can be reduced to a smaller area around the waveguide, see Fig. 1. At the boundary of this optical domain, it is assumed that the magnetic field is zero as the energy density quickly decays outside the waveguide. Thus, the perfectly magnetic conductor boundary condition is applied

$$
e_{i j k} H_{j} m_{k}=0,
$$

where $e_{i j k}$ again is the alternating symbol.

\section{Practical implementation}

The coupled model is solved by the commercial finite element program COMSOL MULTIPHYSICS with MATLAB. ${ }^{17}$ This program is designed for modeling engineering problems described by partial differential equations and it is possible to combine different physical models and solve multiphysics problems. The models can be defined by writing conventional differential equations directly or it is possible to use the application modes, which are templates for specific physical problems with the appropriate equations and variables predefined. In this work the piezoelectric model is implemented in the general form where the governing equations and boundary conditions are written on divergence form. The optical problems are solved using the perpendicular waves, hybrid-mode waves, application mode. The problems are discretized by a triangular element mesh and second order Lagrange elements are employed. A sufficient number of elements must be used in wave problems in order to resolve the waves and obtain convergence, which typically means that at least 5-6 second order elements per wavelength must be employed. In the studied problem, the geometry is complicated and a coupled model is used, so a fine mesh is employed where the wavelength of the vibration in the electrodes for the highest frequency is discretized by at least ten elements. For $a=7 \mu \mathrm{m}$, a maximum element size of $1.5 \mu \mathrm{m}$ is used except in the waveguide area where $0.25 \mu \mathrm{m}$ is used, see Fig. 1. For the piezoelectric model, it has been tested that the results for the phase velocity and the mechanical energy did not change for a finer mesh. For the coupled model, it was furthermore ensured that the values of the refractive indices did not change for a finer mesh and for a bigger optical domain.

\section{RESULTS}

The SAWs generated by HAR electrodes by the finite model are first compared to the results obtained by the periodic model from Ref. 12. The finite model is then employed to calculate the acousto-optical interaction in an optical waveguide.

\section{A. Simulation of SAWs: Comparison between a periodic and a finite model}

The SAWs are generated by Ni electrodes placed on top of the $\mathrm{Y}+128^{\circ}$ cut $\mathrm{LiNbO}_{3}$ substrate where the material matrices are rotated $128^{\circ}$ around the $x_{1}$-axis. Material constants 


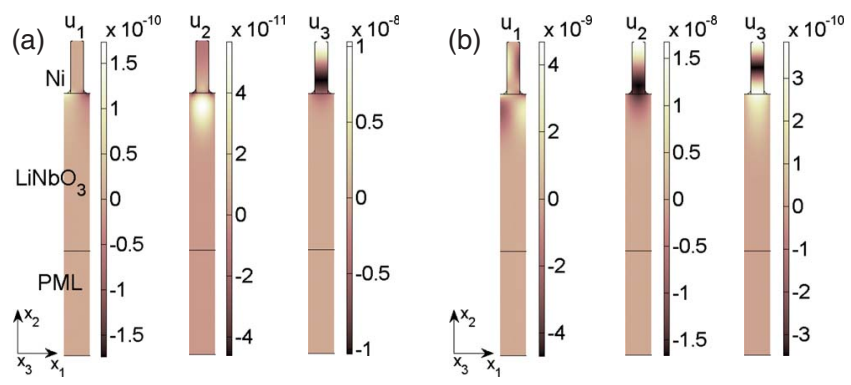

FIG. 2. (Color online) Results for the periodic model where the displacements $u_{1}, u_{2}$, and $u_{3}$ are shown at resonance with the electrode aspect ratio $h / 2 p=1$. (a) Displacements for mode 3 (SH2) for half of the phase velocity $f p=1004 \mathrm{~m} \mathrm{~s}^{-1}$. (b) Displacements for mode 4 (VP2) for $f p=1512 \mathrm{~m} \mathrm{~s}^{-1}$.

for $\mathrm{LiNbO}_{3}$ are taken from Ref. 18. Young's modulus for Ni is $E=200 \mathrm{GPa}$ and Poisson's ratio is $\nu=0.31$. The results for the SAWs obtained by the finite model are first compared to the results found by studying the unit cell with one electrode from Ref. 12. The geometry is illustrated in Fig. 2 where the height of the substrate is $6 p$. A PML is placed at the bottom of the domain with the height $4 p$, and periodic boundary conditions connect the left- and right-hand side boundaries of the substrate. An electrical potential of $1 \mathrm{~V}$ was applied to excite the device and it is found that an increasing number of modes can be excited for increasing aspect ratio and six modes exist for $h / 2 p=1$. Three of the modes are mainly polarized in the shear horizontal (SH) direction and the other three modes are mainly vertically polarized (VP). Examples are given in Fig. 2(a) and 2(b) where the displacements $u_{1}$, $u_{2}$, and $u_{3}$ in the $x_{1^{-}}, x_{2^{-}}$, and $x_{3}$-directions are plotted for modes 3 and 4 at resonance. These plots clearly show that the modes are, in fact, a combination of a vibration in the electrode and a surface wave. It is also verified that mode 3 has a $\mathrm{SH}$ polarization and that mode 4 has a VP polarization, note the different scaling. By plotting the mode shapes, it was found that the electrodes vibrate with modes of increasing order such that mode 3, which is also denoted as $\mathrm{SH} 2$, vibrates in the $x_{3}$-direction with a mode shape of order 2 and mode 4 (VP2) vibrates in the $x_{1}$ - and $x_{2}$-directions with a mode shape of order 2 as well. So it is possible for more and more modes to exist for larger aspect ratio as the electrodes vibrate with modes of higher order. In Ref. 12, the mechanical energy in the structure was calculated, which is the sum of the stored strain and kinetic energy and the mechanical flux flowing into the PML at the bottom. The expressions are given in Ref. 15. The fraction of mechanical energy in the electrode $E_{\text {mech,elec }}$ with respect to the total mechanical energy in the structure $E_{\text {mech,tot }}$ is repeated in Fig. 3(b) for the aspect ratios $h / 2 p \in$ [0.05:3] for the later discussion in Sec. III C. The mechanical energy gets more confined to the electrode for increasing aspect ratio. Thus, the electrode acts as a mechanical resonator, which slows down the SAW velocity because of mechanical energy storage. The modes of the same type tend to the same fraction of mechanical energy in the electrode for increasing aspect ratio because the movement tends to the clean cantilever vibration for slender beams. The only energy left in the substrate is what connects the cantilever modes to the substrate and as modes of the same polarization type deflect in the same direction, the stiff-

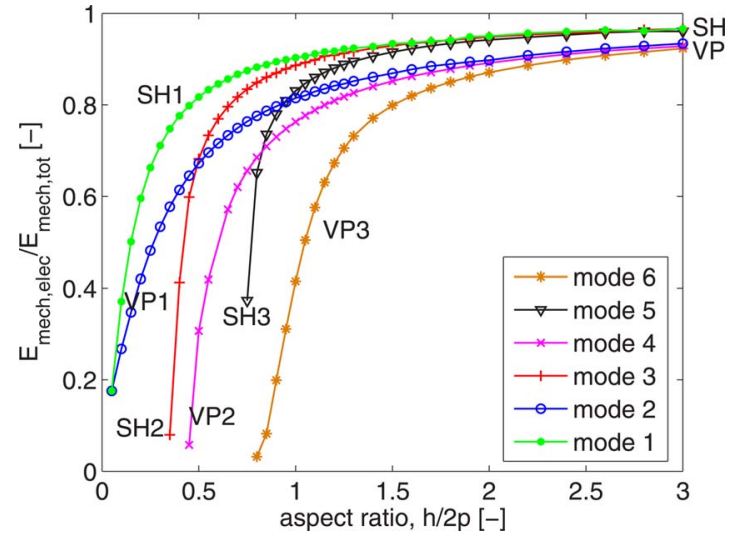

FIG. 3. (Color online) The fraction of mechanical energy in the electrode compared to the total mechanical energy in the structure $E_{\text {mech,elec }} / E_{\text {mech,tot }}$ is plotted as function of the electrode aspect ratio $h / 2 p$ for the six modes computed by the periodic model.

ness in the substrate for these modes is the same. For modes of higher order, the aspect ratio must be bigger before the energy fraction limit is reached, as the wavelength of the vibration is shorter for the same aspect ratio.

Now the wave propagation is studied in the finite device, which is modeled by introducing PMLs at the vertical borders and at the bottom of the substrate, as illustrated in Fig. 1 . The device has 24 electrodes and is excited with alternating potentials of +1 and $-1 \mathrm{~V}$. The aspect ratio $h / 2 p=1$ is chosen for the remaining part of the paper. Two examples of the displacement fields for modes 3 (SH2) and 4 (VP2) at resonance are given in Fig. 4. Mode 3 is the $\mathrm{SH}$ type, so displacement $u_{3}$ is dominant and is plotted in Fig. 4(a). Mode 4 is the VP type, so displacements $u_{1}$ and $u_{2}$ are dominant and $u_{2}$ is shown in Fig. 4(c). Compared to the plots for the periodic model in Fig. 2, it can now be seen how the vibrations are build up and are strongest in the middle. In both cases, a SAW is propagating away from the electrodes and is absorbed in the PMLs, which can just be seen for mode 4 . The two zooms of the area around the electrodes in Figs. 4(b) and 4(d) show that the displacement patterns are the

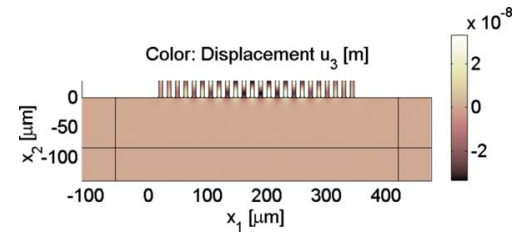

(a)

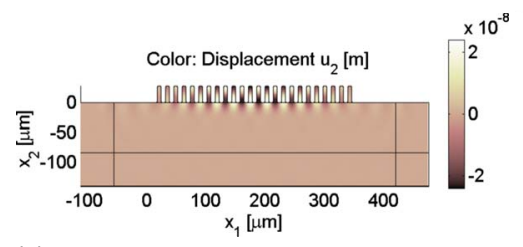

(c)

FIG. 4. (Color online) Results for the finite model at resonance with the electrode aspect ratio $h / 2 p=1$. [(a) and (b)] The out of plane displacements $u_{3}$ for mode 3 (SH2) with half of the phase velocity $f p=1004 \mathrm{~m} \mathrm{~s}^{-1}$. [(c) and (d)] The vertical displacements $u_{2}$ for mode 4 (VP2) with $f p$ $=1510 \mathrm{~m} \mathrm{~s}^{-1}$. 
TABLE I. Half of the phase velocity $f p$ and the fraction of the mechanical energy in the electrodes compared to the total mechanical energy in the structure $E_{\text {mech,elec }} / E_{\text {mech,tot }}$ for the periodic and finite problems for the six modes with electrode aspect ratio $h / 2 p=1$.

\begin{tabular}{|c|c|c|c|c|c|c|c|}
\hline $\begin{array}{l}\text { Mode No. } \\
\text { Mode type }\end{array}$ & & $\begin{array}{c}1 \\
\text { SH1 }\end{array}$ & $\begin{array}{c}2 \\
\text { VP1 }\end{array}$ & $\begin{array}{c}3 \\
\mathrm{SH} 2\end{array}$ & $\begin{array}{c}4 \\
\mathrm{VP} 2\end{array}$ & $\begin{array}{c}5 \\
\text { SH3 }\end{array}$ & $\begin{array}{c}6 \\
\text { VP3 }\end{array}$ \\
\hline \multirow{4}{*}{$\begin{array}{l}\text { phase velocity } \\
f p\left(\mathrm{~m} \mathrm{~s}^{-1}\right) \\
E_{\text {mech,elec }} / E_{\text {mech,tot }}(\%)\end{array}$} & Periodic device & 337 & 523 & 1004 & 1512 & 1641 & 2273 \\
\hline & Finite device & 337 & 523 & 1004 & 1510 & 1639 & 2275 \\
\hline & Periodic device & 90.5 & 81.7 & 88.8 & 76.4 & 83.1 & 41.7 \\
\hline & Finite device & 90.3 & 81.5 & 88.5 & 75.5 & 82.1 & 45.0 \\
\hline
\end{tabular}

same as for the periodic model shown in Figs. 2(a) and 2(b). In order to compare the results for the periodic and the finite model, half of the phase velocity $f p$ for the six modes are given in Table I for both cases, where $f$ represents the resonance frequency. It is seen that the phase velocities are identical for the first three modes and only deviate slightly for the last three modes. So the resonances for the modes are in fine agreement for the periodic and finite models. Finally, the fraction of mechanical energy stored in the electrodes compared to the total mechanical energy in the structure is given in Table I for the periodic and finite cases. All the energy ratios for the finite device are close to the ratios for the periodic device, but they are deviating more with increasing mode number.

These results show that the periodic and finite models appear to be in fine agreement. A tendency is that the values of the phase velocity and the mechanical energy ratio deviate more and more from the periodic device, which can be explained by the mode shapes getting increasingly complicated with increasing mode number, and more energy is lost to bulk waves for the finite structure.

\section{B. Simulation of optical waves}

The results are now presented for the optical eigenvalue problem described in Sec. II C. The optical waveguide is supposed to be created by annealed proton exchange, ${ }^{19}$ and the analytical expression for the refractive index distribution $n\left(x_{1}, x_{2}\right)$ is

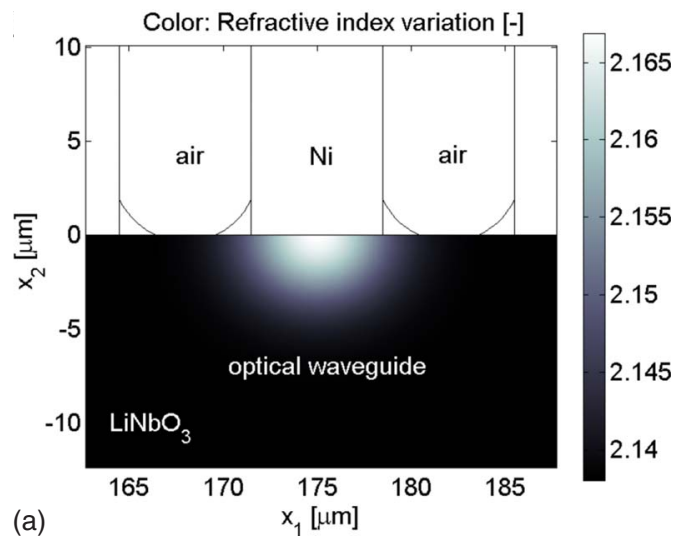

$$
n\left(x_{1}, x_{2}\right)=2.138+0.0289 e^{-\left(0.08 x_{2}^{2}+0.0638\left(x_{1}-175\right)^{2}\right)} .
$$

The unit for $x_{1}$ and $x_{2}$ is microns. The refractive index distribution given by Eq. (18) is illustrated by Fig. 5(a) and it creates a waveguide that can confine an optical mode. The free space wavelength of the optical wave is set to $\lambda_{0}$ $=1.55 \mu \mathrm{m}$-the wavelength used in telecommunication. The refractive index constant for $\mathrm{LiNbO}_{3}$ is $n_{\mathrm{Li}}=2.138$ and the strain-optical constants are found in Ref. 14. The refractive index for $\mathrm{Ni}$ is set to $n_{\mathrm{Ni}}=3.5+6 i$ according to Ref. 20. It is assumed that $n_{\mathrm{Ni}}$ is not changed by the applied strain as the optical mode has almost no energy in the Ni electrode and therefore the acousto-optical modulation has no contribution from the electrode strain. For air simply $n_{\text {air }}=1$ is used. Figure 5(b) shows the $x_{3}$-component of the time averaged power flow of the fundamental mode in the waveguide obtained by solving the eigenvalue problem when no strain is applied (for $\left.V_{p}=0 \mathrm{~V}\right)$. The power flow is confined to the waveguide and the effective refractive index is found to be $n_{\text {eff, } 1}=2.148$ $+7.858 \times 10^{-5} i$. The real part is between the highest value of the refractive index in the waveguide and the value in the substrate, as expected. The small imaginary part is the attenuation and has a value because of the imaginary part in the refractive index in the $\mathrm{Ni}$ electrodes that absorb energy from the system. The fundamental mode is mainly polarized in the $x_{1}$-direction. The waveguide supports several modes and the next mode is also a first order mode with a similar mode shape. It is mainly polarized in the $x_{2}$-direction with the effective refractive index $n_{\text {eff }, 2}=2.147+6.090 \times 10^{-4} i$. In this paper, we are only interested in the acousto-optical in-

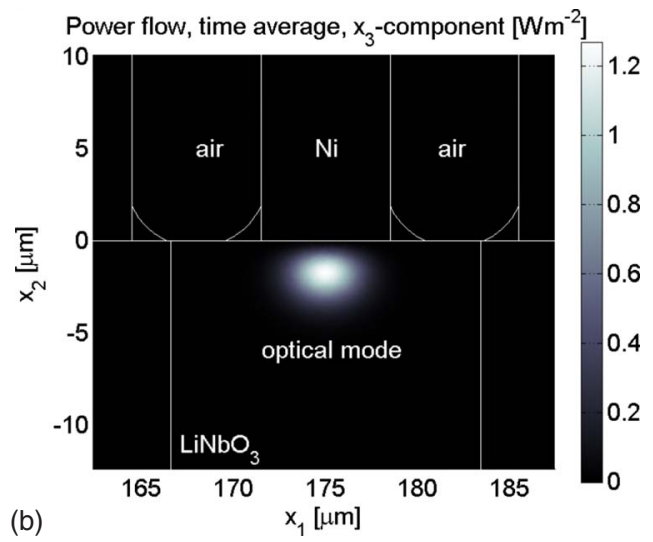

FIG. 5. (Color online) Results for the optical model with the electrode aspect ratio $h / 2 p=1$. (a) The refractive index variation that defines the waveguide. (b) The distribution of the $x_{3}$-component of the time averaged power flow for the fundamental mode in the waveguide with arbitrary scale when no strain is applied. 
TABLE II. The difference in effective refractive index normalized with the squared applied electrical power for the two first order optical modes for each of the six acoustic modes with electrode aspect ratio $h / 2 p=1$ as well as for the thin electrodes with $h / 2 p=0.01$.

\begin{tabular}{lcc}
\hline \hline & $\begin{array}{c}\Delta n_{\text {eff, } 1 / \sqrt{P}} \\
\left(\mathrm{~W}^{-1 / 2}\right)\end{array}$ & $\begin{array}{c}\Delta n_{\text {eff, } 2 / \sqrt{P}} \\
\left(\mathrm{~W}^{-1 / 2}\right)\end{array}$ \\
\hline Mode 1 (SH1) & $1.55 \times 10^{-3}$ & $8.97 \times 10^{-4}$ \\
Mode 3 (SH2) & $9.08 \times 10^{-5}$ & $6.62 \times 10^{-5}$ \\
Mode 5 (SH3) & $3.08 \times 10^{-6}$ & $2.50 \times 10^{-6}$ \\
Mode 2 (VP1) & $7.35 \times 10^{-5}$ & $7.21 \times 10^{-4}$ \\
Mode 4 (VP2) & $1.18 \times 10^{-5}$ & $6.35 \times 10^{-5}$ \\
Mode 6 (VP3) & $6.49 \times 10^{-6}$ & $1.38 \times 10^{-5}$ \\
Thin electrodes & $2.51 \times 10^{-6}$ & $1.71 \times 10^{-6}$ \\
\hline \hline
\end{tabular}

teraction between the acoustic and optical waves, so in the following the imaginary part of the effective refractive indices is not included. In practice, this approach is justified if the interaction length is less than 100 optical wavelengths. For longer interaction lengths, attenuation could become significant and should be included in the analysis.

\section{Improving the acousto-optical interaction}

The coupled acousto-optical model is now solved for each of the six acoustic modes for the case where a wave crest is at the waveguide and a wave trough is at the waveguide, respectively. The change between the two extreme cases is given as

$$
\Delta n_{\mathrm{eff}, j}=\left|n_{\mathrm{eff}, j}^{\mathrm{c}}-n_{\mathrm{eff}, j}^{\mathrm{t}}\right|
$$

where $j$ is 1 for the mode that is polarized in the $x_{1}$-direction and 2 for the mode polarized in the $x_{2}$-direction. The modes will be denoted as optical modes 1 and 2 . The superscript $\mathrm{c}$ in Eq. (19) indicates the value at a wave crest and $t$ indicates the value at a trough. The difference $\Delta n_{\text {eff }, j}$ is normalized to the square root of the time averaged applied electric power $P$ calculated by the following expression found in Ref. 15:

$$
P=\Re \int_{\mathrm{L}_{\mathrm{el}}} \frac{V\left(i \omega D_{2} m_{2}\right)^{*}}{2} \mathrm{dL},
$$

where $\mathrm{L}_{\mathrm{el}}$ corresponds to the interfaces between the electrodes and the substrate and $m_{2}$ is the normal vector to these interfaces. The star indicates the complex conjugate. The results are reported in Table II for the six acoustic modes and for thin electrodes with $h / 2 p=0.01$ for both optical modes 1 and 2. The tendency is that the SH modes interact most efficiently with optical mode 1 and the VP modes interact best with optical mode 2. The acousto-optical interaction decreases with increasing mode number within the two different categories of the acoustic modes. The six acoustic modes with HAR electrodes interact better in general compared to the acoustic mode for the conventional thin electrodes. The interaction is a result of how efficiently the acoustic mode is excited with the applied electrical power and how well the acoustic and optical modes match each other. The calculations show that the acoustic modes of lower order are more efficiently excited by a certain amount of electrical power than the modes of higher order such that larger values of the strain and hence bigger change in refractive index for lower order modes are obtained. A reason for this tendency is that for higher aspect ratios, the movement of the structure tends to clean cantilever vibrations with the mechanical energy concentrated in the electrodes and just below them where they are attached to the substrate. The graphs in Fig. 3 show that the lower order acoustic modes are closer to this limit for $h / 2 p=1$ and therefore more strain is concentrated in the area just below the electrodes. As the structure with HAR electrodes is more compliant than the almost plane surface with the thin electrodes, more power has to be applied to the thin electrode in order to get the same strain. It can therefore be expected that the change in refractive index is biggest for the low order acoustic modes with HAR electrodes.

The other important question is how well the acoustic modes match with the optical modes, in other words how well the changes in refractive index and the optical modes overlap. The optical modes will mainly detect the changes in refractive index in their polarization direction. So for optical mode 1 , it is the SAW induced changes of the refractive index in the horizontal direction $\Delta n_{11}$ that are important. Optical mode 2 must overlap with $\Delta n_{22}$. The acoustic modes of the same polarization type all have a similar pattern of changes in refractive index around the optical waveguide. As an example, $\Delta n_{11} / \sqrt{P}$ is plotted for SH1 in Fig. 6(a) with the power flow of optical mode 1 indicated with contour lines. $\Delta n_{22} / \sqrt{P}$ is plotted with optical mode 2 in Fig. 6(b). When these two figures are compared, it is seen that $\Delta n_{11} / \sqrt{P}$ has bigger values than $\Delta n_{22} / \sqrt{P}$, but the distribution of these parameters have a similar pattern that both match the optical modes well. This is a tendency for all the SH modes. However, for SH modes of higher order, the wave patterns tend to go deeper into the substrate and therefore the strain concentrations are not as big around the waveguide. The same is plotted for VP1 in Figs. 6(c) and 6(d), note the different color scales. In this case $\Delta n_{22} / \sqrt{P}$ has the biggest values and the distribution overlaps well with the optical mode. $\Delta n_{11} / \sqrt{P}$ does not overlap so well, as it has a pattern where $\Delta n_{11} / \sqrt{P}$ changes sign into the depth. A part of optical mode 1 is therefore overlapping with $\Delta n_{11} / \sqrt{P}$ being close to zero. This is also a tendency for all the VP modes. Thus, the mode patterns explain that the interaction is different for the $\mathrm{SH}$ and VP modes with respect to the polarization direction of the optical mode. The interaction is most efficient for SH1 with optical mode 1 and it is more than 600 times bigger than for the case with the thin electrodes. In Figs. 7(a) and $7\left(\right.$ b), the change in refractive indices $\Delta n_{11} / \sqrt{P}$ and $\Delta n_{22} / \sqrt{P}$ are plotted for the case with thin electrodes, respectively. It is seen that the changes are small compared to the acoustic modes SH1 and VP1 in Fig. 6 and that the overlap with the optical modes is not as good. In the case with the overlap between $\Delta n_{22} / \sqrt{P}$ and optical mode 2 , the center of the optical mode is where the change in refractive index is zero.

The results show that it is possible to get a more efficient acousto-optical interaction with the new types of SAWs compared to using conventional IDTs, where Rayleigh-type waves are generated. It is important to know how well the acoustic mode is excited for the applied electrical power in order to get bigger changes in refractive index. The distribu- 

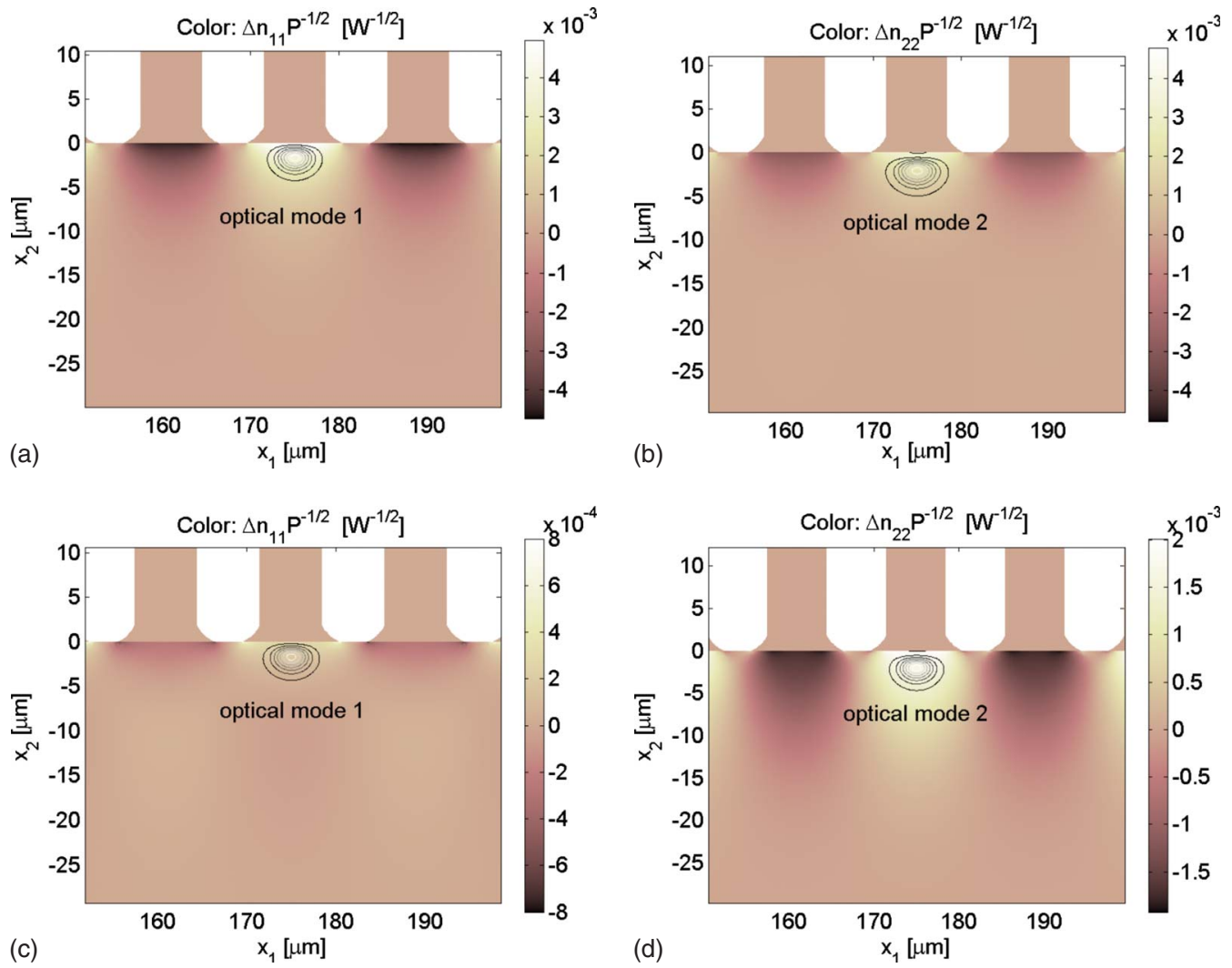

FIG. 6. (Color online) Results for the acousto-optical interaction with electrode aspect ratio $h / 2 p=1$. The color scales indicate the refractive index change $\Delta n_{11}$ or $\Delta n_{22}$ normalized with the square root of the applied electrical power $P$. The contour lines illustrate the out of plane time-average power flow of the optical modes with arbitrary scale. (a) Acoustic mode 1 (SH1) and optical mode 1. (b) Acoustic mode 1 (SH1) and optical mode 2. (c) Acoustic mode 2 (VP1) and optical mode 1. (d) Acoustic mode 2 (VP1) and optical mode 2.

tion of the change in refractive index in the different directions must match an optical mode polarized in a certain direction. In this work, the SH modes overlap the horizontally polarized optical mode best and the VP modes match best with the optical mode, which is VP. The stronger modulation of the optical waves could improve the modulation efficiency in structures like acousto-optical multiple interference devices. The concept of these devices are presented in Ref. 21 where several MZIs are combined in parallel or series in order to design on/off switching, pulse shapers, and frequency converters.

\section{CONCLUSION AND FURTHER WORK}

In this paper, SAWs are generated by HAR electrodes and the interaction with optical waves in a waveguide is studied in order to improve integrated acousto-optical modulators. It is explained how the SAWs can be generated by HAR electrodes by employing a 2D model of a piezoelectric anisotropic material where reflections from the boundaries are avoided by PMLs. This model is then coupled to a model of the optical wave such that the change in effective refractive index introduced in the waveguide by the strain from the
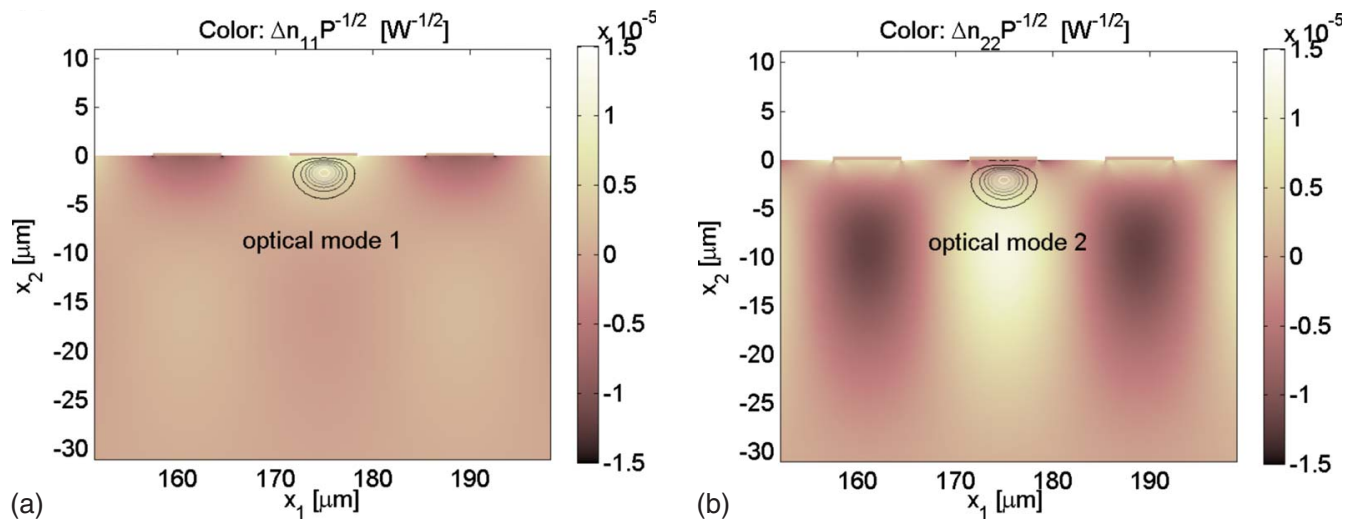

FIG. 7. (Color online) Results for the acousto-optical interaction with electrode aspect ratio $h / 2 p=0.01$. The color scales indicate the refractive index change $\Delta n_{11}$ or $\Delta n_{22}$ normalized with the square root of the applied electrical power $P$. The contour lines illustrate the out of plane time-average power flow of the optical modes with arbitrary scale. (a) Optical mode 1. (b) Optical mode 2. 
SAWs can be calculated.

A finite model with 12 electrode pairs is considered and 6 different acoustic modes can be generated for the chosen aspect ratio of the electrodes. A fine agreement for the mode shapes, the resonance frequencies, and the mechanical energy confinement to the electrodes is found between the finite model and the periodic model studied in Ref. 12. The finite model is then employed to study the acousto-optical interaction in a waveguide. The optical modes in the waveguide are found by solving the time-harmonic wave equation. By applying strain from the six acoustic modes for the case where a wave crest is at the waveguide and a trough is at the waveguide, the difference in effective refractive index normalized with the square root of the applied electrical power is found for the two first order optical modes. The efficiency of the interaction depends on how well the acoustic mode is excited and how good the acoustic and the optical modes overlap. The interaction decreases with increasing number of acoustic mode, and the modes polarized in the $\mathrm{SH}$ direction interact best with the optical wave polarized in the horizontal direction and the vertical polarized acoustic modes interact best with the vertical polarized optical mode. Compared to the case with the conventional thin electrodes, the acousto-optical interaction can be increased more than 600 times with the new type of SAWs.

Further work includes a study of the optimal position and size of the waveguide compared to the electrode size. Also the optimal aspect ratio for each of the six modes can be explored, and it is expected that the interaction will increase until the limit of the mechanical energy fraction has been reached as the mechanical energy will be more concentrated around the electrodes and less power has to be applied to obtain certain strain values.

\section{ACKNOWLEDGMENTS}

This work is supported by the European FP6 research project ePIXnet-European Network of Excellence on Photonic Integrated Components and Circuits. The authors are grateful to Ole Sigmund and Jakob S. Jensen from the Department of Mechanical Engineering and Martin P. Bendsøe from the Department of Mathematics, Technical University of Denmark, for helpful discussions related to the presented work. The support from Euro-horcs/ESF European Young Investigator Award (EURYI) through the grant Synthesis and topology optimization of optomechanical systems as well as the Danish Center for Scientific Computing is gratefully acknowledged.

${ }^{1}$ K.-Y. Hashimoto, Surface Acoustic Wave Devices in Telecommunications, Modelling and Simulation (Springer, Berlin, 2000).

${ }^{2}$ S. Takahashi, H. Hirano, T. Kodama, F. Miyashiro, B. Suzuki, A. Onoe, T. Adachi, and K. Fujinuma, IEEE Trans. Consum. Electron. CE-24, 337 (1978).

${ }^{3}$ Proceedings of the International Symposium on Surface Acoustic Wave Devices for Mobile Communication, 1992, edited by K. Shibayama and K. Fujinuma (unpublished).

${ }^{4}$ U. Wolff, F. L. Dickert, G. K. Fischauer, W. Greibl, and C. C. W. Ruppel, IEEE Sens. J. 1, 4 (2001).

${ }^{5}$ M. M. de Lima, Jr. and P. V. Santos, Rep. Prog. Phys. 68, 1639 (2005).

${ }^{6}$ C. Gorecki, F. Chollet, E. Bonnotte, and H. Kawakatsu, Opt. Lett. 22, 1784 (1997).

${ }^{7}$ M. M. de Lima, Jr., M. Beck, R. Hey, and P. V. Santos, Appl. Phys. Lett. 89, 121104 (2006).

${ }^{8}$ M. van der Poel, M. Beck, M. B. Dühring, M. M. de Lima, Jr., L. H. Frandsen, C. Peucheret, O. Sigmund, U. Jahn, J. M. Hwam, and P. V. Santos, Proceedings of the European Conference on Integrated Optics and Technical Exhibition, Copenhagen, Denmark, 25-27 April 2007 (unpublished).

${ }^{9}$ M. B. Dühring and O. Sigmund, J. Appl. Phys. 105, 083529 (2009).

${ }^{10}$ V. Laude, A. Khelif, T. Pastureaud, and S. Ballandras, J. Appl. Phys. 90, 2492 (2001).

${ }^{11}$ V. Laude, L. Robert, W. Daniau, A. Khelif, and S. Ballandras, Appl. Phys. Lett. 89, 083515 (2006).

${ }^{12}$ M. B. Dühring, V. Laude, and A. Khelif, J. Appl. Phys. 105, 093504 (2009).

${ }^{13}$ U. Basu and A. Chopra, Comput. Methods Appl. Mech. Eng. 192, 1337 (2003).

${ }^{14}$ D. Royer and E. Dieulesaint, Elastic Waves in Solids, 1st ed. (Springer, New York, 2000).

${ }^{15}$ B. A. Auld, Acoustic Fields and Waves in Solids, 1st ed. (Wiley, New York, 1973).

${ }^{16}$ R. Syms and J. Cozens, Optical Guided Waves and Devices, 1st ed. (McGraw-Hill, New York, 1992).

${ }^{17}$ COMSOL Reference Manual for COMSOL 3.3A, COMSOL AB, Stockholm, www.comsol.se.

${ }^{18}$ G. Kovacs, M. Anhorn, H. E. Engan, G. Visintini, and C. C. W. Ruppel, Proc.-IEEE Ultrason. Symp. 1, 435 (1990).

${ }^{19}$ M. L. Bortz and M. M. Fejer, Opt. Lett. 16, 1844 (1991).

${ }^{20}$ M. A. Ordal, R. J. Bell, R. W. Alexander, L. L. Long, and M. R. Querry, Appl. Opt. 24, 4493 (1985).

${ }^{21}$ M. Beck, M. M. de Lima, Jr., and P. V. Santos, J. Appl. Phys. 103, 014505 (2008). 